\title{
Novel CFD-Based Full-Scale Resistance Prediction for Large Medium-Speed Catamarans
}

\author{
Max Haase $^{\mathrm{a}, *}$, Konrad Zürcher ${ }^{\mathrm{a}}$, Gary Davidson ${ }^{\mathrm{b}}$, Jonathan Binns ${ }^{\mathrm{a}}$, Giles \\ Thomas $^{\mathrm{c}}$, Neil Bose ${ }^{\mathrm{a}}$ \\ ${ }^{a}$ Australian Maritime College, University of Tasmania, Australia \\ ${ }^{b}$ Revolution Design / Incat Tasmania, Australia \\ ${ }^{c}$ Department of Mechanical Engineering, University College London, UK
}

\begin{abstract}
A novel CFD-based approach is presented that is used in conjunction with model test experiments to predict ship resistance at full-scale Reynolds and Froude numbers. It relies on verification using model scale experiments, including an agreement of integrated shear force with established model-ship correlation lines at model and full-scale, and includes surface roughness effects. One major advantage of the method is that the geometric dimensions of the CFD modelling remain at model scale. CFD simulation results were successfully verified considering the drag of two different catamarans at 1:22 and 1:50 model scale. Furthermore, it is shown that an identical near-wall mesh can be used for both model and full-scale simulations without compromising the accuracy of the shear force. At full-scale the deviation of resistance between CFD prediction, model test extrapolation and full-scale measurements of a 98 m catamaran was as low as $5 \%$ at $F \mathrm{r}=0.40$ and 0.43 . For a novel $130 \mathrm{~m}$ catamaran variations in full-scale drag for a smooth hull were also less than $5 \%$ when comparing extrapolated model scale experiments and CFD predictions. However, at such large Reynolds numbers CFD predictions for correlation and roughness allowance were significantly higher compared to estimates proposed in ITTC guidelines.
\end{abstract}

Keywords: full-scale CFD, medium-speed catamarans, resistance prediction

*Corresponding author: max.haase@utas.edu.au 


\section{Introduction}

Large medium-speed catamarans are evolving from current high-speed catamarans as a fuel-efficient alternative to satisfy requirements for environmentally sustainable and economic efficient fast sea transportation. In previous studies

5 the authors have shown that for fast catamarans the transport efficiency, which can be understood as the payload per installed engine power at a certain speed, can be significantly increased due to increasing size and reduced speed $[1,2]$. An image of this novel type of ship is presented in Figure 1. However, due to the novelty of this vessel type, experience in applying appropriate resistance prediction methods is lacking and therefore in the preliminary design phase of such vessels it is not currently possible for designers to confidently predict their resistance and hence powering requirements. A novel approach based on computational fluid dynamics aided by model test experiments has been developed and is presented here. It has been validated by successfully comparing results with predictions from extrapolated model test data and results from full-scale sea trials.

For a new class of ship there are several reasons why the accuracy of predicted full-scale resistance values, that are based on extrapolated model test data using the procedures established by the International Towing Tank Conference (ITTC), should be questioned. Particularly since no model-ship correlation data will be available which is essential as the extrapolation process is currently always based on empirical input [3].

Firstly, whilst recommended procedures and guidelines for full-scale resistance prediction based on model test extrapolation have been established by the ITTC for conventional (7.5-02-03-01.4) and high-speed craft (7.5-02-05-01), they do not include explicit details on an approach for medium-speed catamarans. Even though medium-speed catamarans will operate at speeds of $20-35$ knots, which are typical for conventional monohull ferries, their slender demihulls in close proximity of each other are rather a feature of high-speed craft. 
compared to contemporary high-speed catamarans. The demihulls of mediumspeed vessels have more convex buttock lines and significantly reduced transom immersion to account for operating around hump speed, where wave-making usually dominates the overall resistance [2]. Therefore they cannot be clearly 35 categorised as either high-speed craft nor conventional vessels.

Secondly, in the conventional resistance extrapolation approach of ITTC (7.5-02-03-01.4), the total drag force is decomposed into the frictional component $C_{F} \times(1+k)$ and a residuary component $C_{R}$. $C_{F}$ solely depends on Reynolds number, $(1+k)$ is a function of hull form geometry and $C_{R}$ depends on Froude 40 number and hull form geometry. The first two are assumed to be independent of the Froude number, while the latter two are assumed to be independent of hull size and Reynolds number. However, in previous research [4], it was found that at medium speeds the Froude number also has an influence on the vessels shear force. This can be understood as a form effect due to changes in the running attitude and waterline around the vessel. Traditionally the form effect is accounted for by the form factor $(1+k)$, which is estimated at $F r \rightarrow 0$. A large bow down trim is required to assure the dry transom condition is achieved to estimate the form factor according to ITTC guidelines. However, this corresponds to an impractical floating condition, which is an unrealistic ship operation and may lead 50 to inapplicable values of $(1+k)$. Alternatively, an empirically determined value for $(1+k)$ may be chosen [5]. Furthermore, an effect of Reynolds number on the Froude dependent part of the total resistance is also expected, because the pressure recovery at the stern is potentially influenced by the boundary layer thickness.

Thirdly, to predict full-scale resistance from model test experiments either an experience-based correlation allowance is added that takes the scale factor, hull surface roughness, ship type and other scaling effects into account. Alternatively, an empirical allowance can be estimated for conventional ships, which according to ITTC guidelines is based on surface roughness, ship length and ${ }_{60}$ full-scale Reynolds number. For this novel type of vessel it is assumed that the available data for the experience-based determination of factors for model-ship 
correlation will be insufficiently accurate, because the ships are significantly different in hull form and size when compared to current fast ferries or high-speed catamarans.

Finally, the ITTC recommended procedures imply that the total resistance coefficient $\left(C_{T s}\right)$ is linearly decomposed. So that the same form factor $(1+k)$ is assumed to be valid for all Froude numbers, that the residuary resistance coefficient $\left(C_{R}\right)$ solely depends on Froude number and will not change with increasing Reynolds number and simply extra drag due to surface roughness $\left(d C_{F}\right)$ or experience-based allowance $\left(C_{A A}\right)$ can be added.

$$
C_{T s}=(1+k) \times C_{F}+C_{R m}+C_{A A}+d C_{F}
$$

where it is assumed that the terms depend on:

$$
\begin{gathered}
(1+k)=f(\text { hull form }) \\
C_{F}=f(\text { Re }) \\
C_{R}=f(\text { Fr, hull form }) \\
d C_{F}=f(\text { surface roughness, ship length }, \text { Re }) \\
C_{A A}=f(\text { Re or experience })
\end{gathered}
$$

These four attributes of the ITTC-based extrapolation method emphasise the need for a tool to predict the full-scale resistance of novel surface vessel and for medium-speed catamarans in particular. An approach based on using computational fluid dynamics (CFD) is instead proposed. [6] showed that CFD can be used to predict the ship performance at both model-scale and full-scale and that a difference in drag force compared to ITTC methods exists which is usually overcome by an empirical correlation allowance. [7] highlighted that the consideration of varying Reynolds number is a key advantage for CFD in full-scale powering prediction. However, validation of full-scale simulation may so be difficult [3] due to spatial requirements of the testing facilities and experience in its applicability is currently insufficient. 


\subsection{Scope of study}

This study introduces an approach to run numerical simulations based on Reynolds-Averaged Navier-Stokes (RANS) equations at full-scale Froude and 85 Reynolds numbers using meshes whose spatial resolution has been verified at model scale. The shear force on smooth and rough flat plates was verified over a large range of Reynolds numbers to derive near-hull mesh parameters applicable for accurate simulations at model and full scale. Full-scale results were validated by comparing them to extrapolated model test data and resistance derived from 90 power measurements of full-scale sea trials of a $98 \mathrm{~m}$ catamaran shown in Figure 2 .

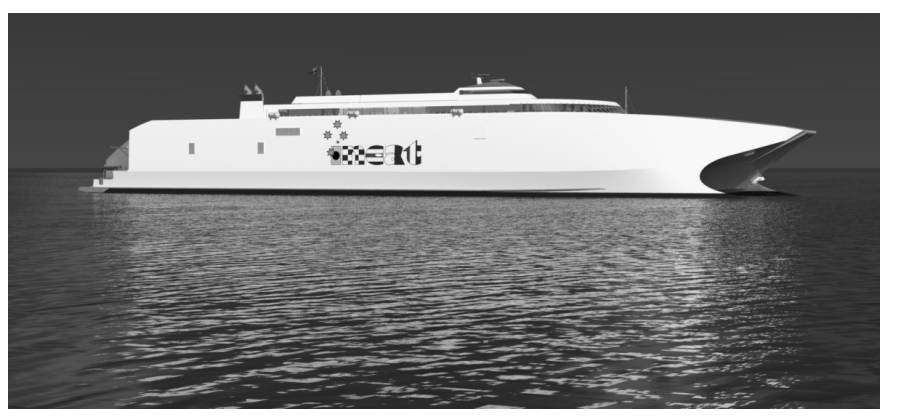

Figure 1: Proposed design of a $130 \mathrm{~m}$ medium-speed catamaran.

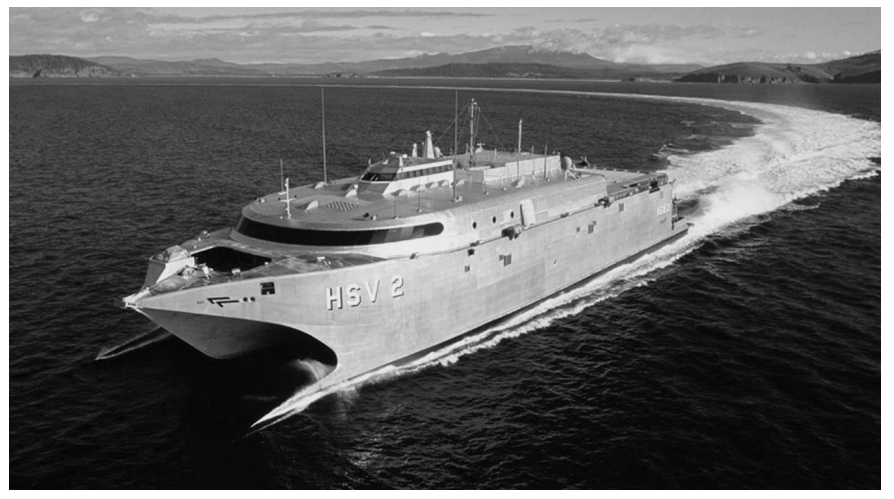

Figure 2: Image of the $98 \mathrm{~m}$ INCAT high-speed catamaran. 


\section{Full-scale CFD approach}

CFD has been applied to full-scale ships scale ships including large highspeed catamarans [8]. However, these results can be only directly validated when comparing to the drag force obtained for the full-scale ship. Especially for large and fast vessels it is considered as being impossible to conduct full-scale towing test and alternatively the drag force has to be derived from self propelled sea trial measurements [9]. Therefore, the novel full-scale CFD approach incorporates model test experiments to verify a spatial resolution of the flow domain for a sufficiently accurate simulation. Also, the method obeys conformity with established model-ship correlation or friction lines to conclude validity of the drag force obtained for the full-scale vessel.

Before conducting simulations for the vessel at full-scale Reynolds numbers, the calm water resistance of a scale model of the ship needs to be obtained through both numerical simulations and physical model test experiments at an identical model scale factor $(\lambda)$. The simulation procedure can be considered as being verified if the resulting total resistance, and sinkage and trim agree with the experimentally measured values. Furthermore it is required that the integral value of shear force over the wetted surface area is in agreement with established model-ship correlation lines $\left(C_{V}=C_{F}\right)$. If the above agreements can be achieved, it is assumed that the flow around the vessel and its physical effects on the hull are sufficiently accurately resolved and pressure and shear force both correctly predicted at model-scale.

Then the Reynolds number is changed to its full-scale value by altering the viscosity of the fluid. It is assumed that the accuracy of the pressure drag solely depends on the spatial domain resolution and is independent of Reynolds number. Without altering the linear dimensions, flow velocity or spatial resolution of the initial mesh, a simulation at full-scale Froude and Reynolds number can now be conducted. The fluid density remains unchanged and the vessels displacement is kept constant. If again an agreement of the shear force coefficient with values from established model-ship correlation lines is found $\left(C_{V}=C_{F}\right)$ 
and the pressure drag is close to the value predicted at model-scale Reynolds number, it was assumed that the results are physically adequate. If required, the simulation can be continued including a certain surface roughness applied to the ship hull to include the effect of paint and marine growth on the total resistance force. With the simulation conducted at full-scale Reynolds and Froude numbers the final results are readily applicable for the full-scale ship when treated non-dimensionally. However, since they are geometrically at model-scale the results need to be multiplied by $\lambda^{3}$ and a relative change in density $\left(\rho_{S W} / \rho_{F W}\right)$ to obtain the dimensional drag force for the full-scale ship. Figure 3 summarises the numerical extrapolation process in a flow chart.

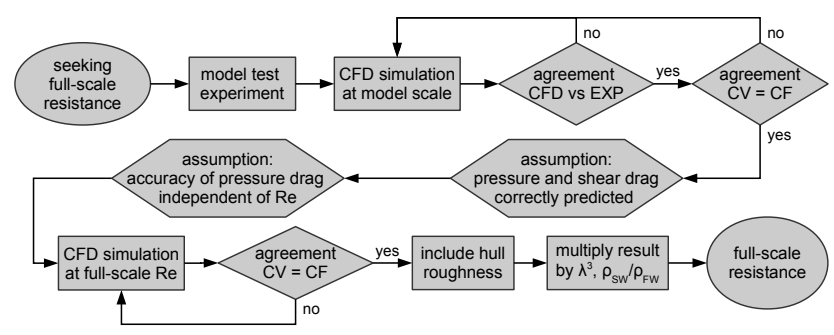

Figure 3: Flowchart to obtain full-scale resistance using computational grids verified at model scale. Top row contains simulations at model scale, middle row shows the assumptions made and bottom row contains simulations at fullscale Reynolds numbers.

Compared to the ITTC procedure, the numerical approach decomposes the resistance into normal $\left(C_{P}\right)$ and tangential stresses $\left(C_{V}\right)$ whereas both can freely change with changing Froude number, Reynolds number and hull form:

$$
\begin{gathered}
C_{T}=C_{P}+C_{V} \\
C_{P}, C_{V}=f(F r, \text { Re, hull form })
\end{gathered}
$$

Furthermore, empirical corrections for surface roughness, finite water effects or form effects are not necessary as they are inherently included in the numerical 
model when solving the Reynolds-Averaged Navier-Stokes equations for the flow around the ship hull.

\subsection{Numerical simulation tool}

The transient solver interDyMFoam of the OpenFOAM CFD toolbox (version 2.3) was used for solving the RANS equations in this study unless stated otherwise. It features viscous free-surface flows and 6 degree of freedom motion to allow dynamic trim and sinkage of the vessel. $k-\omega-S S T$ (shear stress transport) turbulence model and standard wall functions were used throughout. The first cell height was chosen according to the results from Section 4 and set to values around $y_{1}=0.6 \times L \times 10^{-3}$ for both model and full-scale simulations. The computational domain was discretised using the OpenFOAM toolbox meshing tools, including blockMesh, snappyHexMesh and refineMesh. The resulting mesh sizes varied between $700 \mathrm{k}$ - 900k cells when replicating towing tank dimensions.

\subsection{Verification and validation}

Before conducting CFD simulations at full-scale Reynolds numbers, the computational set-up was verified by using model-scale experiments to assure an adequate spatial resolution of the computational domain at model scale. In the current research the verification is based on towing tank experiments of a 1:50 $130 \mathrm{~m}$ medium-speed [1] and 1:22 $98 \mathrm{~m}$ high-speed catamaran [10] at Froude numbers of $\mathrm{Fr}=0.20 .5$. Results are presented in section 3 (Verification of CFD Simulation). The capability of accurately resolving boundary layer flows including surface roughness over a wide range of Reynolds numbers $(6.5<\log (R e)<9.75)$ is demonstrated in section 4 (Verification of near-wall flow). Finally, the full-scale drag value of the $98 \mathrm{~m}$ catamaran was validated based on power measurements from sea trials and furthermore, CFD predictions for both vessels were validated based on extrapolated model test data following ITTC guidelines (7.5-02-03-01.4). Results are presented in section 5 (Validation of full-scale drag). 


\section{Verification of CFD simulations}

Towing tank results of a 1:50 scale model of a $130 \mathrm{~m}$ medium-speed catamaran [1] at demihull displacement conditions corresponding to $L / \nabla^{1 / 3}=11.7$ and results of a $98 \mathrm{~m}$ catamaran at 1:22 model scale by Zrcher [10] were used to verify the accuracy of the CFD simulations. For the latter the displacement was in accordance to a demihull slenderness of $L / \nabla^{1 / 3}=10.2$. Due to width restrictions in the towing tank only half of the hull was modelled for the $98 \mathrm{~m}$ vessel [11] and consequently a single demihull was tested in close proximity to the tank wall where reflecting waves account for the hydrodynamic interaction of the opposing demihulls. The distance of the demihull to the wall equalled half the demihull separation distance, an approach that was successfully utilised previously by Rovere [12].

\subsection{Mesh sensitivity study}

The influence of the cell count on the resistance was studied using the $130 \mathrm{~m}$ medium-speed catamaran. Computational meshes of different spatial resolution ranging from $660 \mathrm{k}$ to $1.3 \mathrm{M}$ cells were investigated. When compared to the results for the finest mesh, the drag force obtained from the coarsest mesh deviated less than $1 \%$ for $F r=0.37$ and less than $2.5 \%$ for $F r=0.45$, and the medium sized mesh differed by no more than $0.5 \%$ at both speeds. In an earlier study [8] it was shown that accurate predictions can be made for catamarans at medium speeds over a wide range of Froude and Reynolds numbers using comparable mesh sizes to the ones used in the current study. The same meshing routine has been used for both vessels as their principal hull form features are similar.

\subsection{Presentation of results}

Comparisons of resistance determined by CFD and model test experiments are presented in Figure 4 and 6. While results from model test experiments are presented using hollow markers, where those of subsequent speeds were connected with thin lines for better readability, CFD results are shown by vertical 
pressure contribution $\left(R_{P}\right)$ and one from tangential stresses $\left(R_{V}\right)$. In contrast, the experimentally determined drag force $\left(R_{T}\right)$ was decomposed into residuary resistance $\left(R_{R}\right)$ and frictional resistance $\left(R_{F}\right)$ as estimated by using the ITTC model-ship correlation line. Resistance force, including its subdivisions, was non-dimensionalised by displacement, density, and gravity and further divided by Froude number squared.

\subsection{Model-scale results of $98 \mathrm{~m}$ catamaran}

The 1:22 scale model of the $98 \mathrm{~m}$ vessel was simulated at $F r=0.2,0.31$, 0.40 and 0.43 which corresponds to Reynolds numbers of $\log (R e)=6.87 .1$. The results of numerical resistance predictions and model scale measurements are shown in Figure 4 where CFD under-estimated the total drag force by less than $5 \%, 8 \%, 8 \%$ and $2 \%$ for the respective speeds. The shear force over the wetted hull deviated by less than $10 \%$ with that predicted by ITTC friction line, where a difference from that line was assumed to result from changing flow around the hull due to wave making and sinkage and trim rather than uncertainties in the prediction methodology. The deep transom of the vessel leads to a major resistance hump around $F r=0.3$ that is not due to the interference of bow and stern wave systems, but rather is related to transom drag. Figure 5 shows that sinkage and trim predicted by the CFD and model test experiments show similar trends. However, the numerically predicted trim is up to $30 \%$ larger than the value measured in experiments and sinkage is up to twice as large as that found in the model scale experiments for $F r \leq 0.4$. It should be noted that the towing tank length was a critical factor during data acquisition, because unsteady resistance effects occurred. ITTC guidelines (7.5-02-02-01) recommend to run the model for at least 5 cycles of those oscillations. However, due to the current set-up the number of recorded cycles was 3 for $\mathrm{Fr}=0.31$ and only half a cycle was resolved for $F r=0.43$. Therefore, deviations between fully converged solution from CFD simulations and model test experiments were expected. 


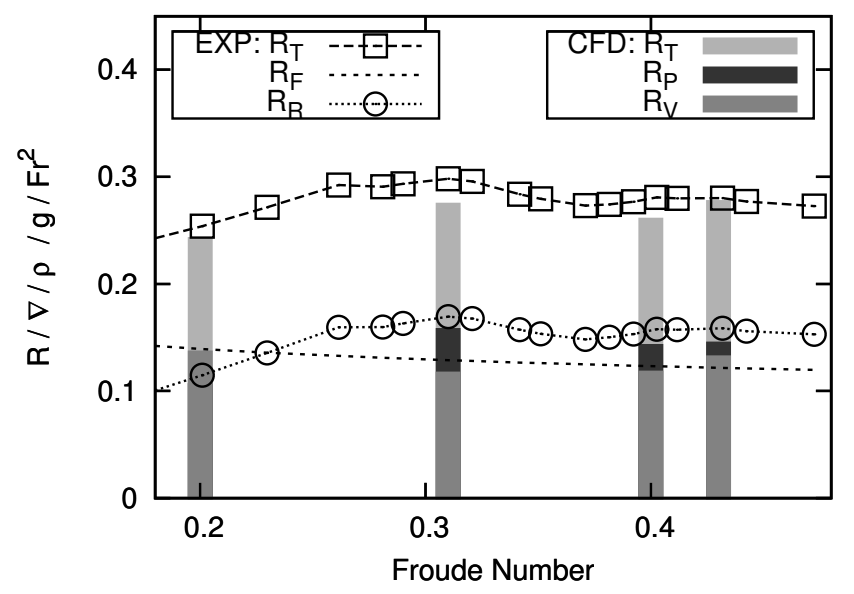

Figure 4: Non-dimensional resistance for $98 \mathrm{~m}$ high-speed catamaran at 1:22 model scale determined by CFD (shown as vertical bars) and physical towing tank experiments (shown as hollow markers).

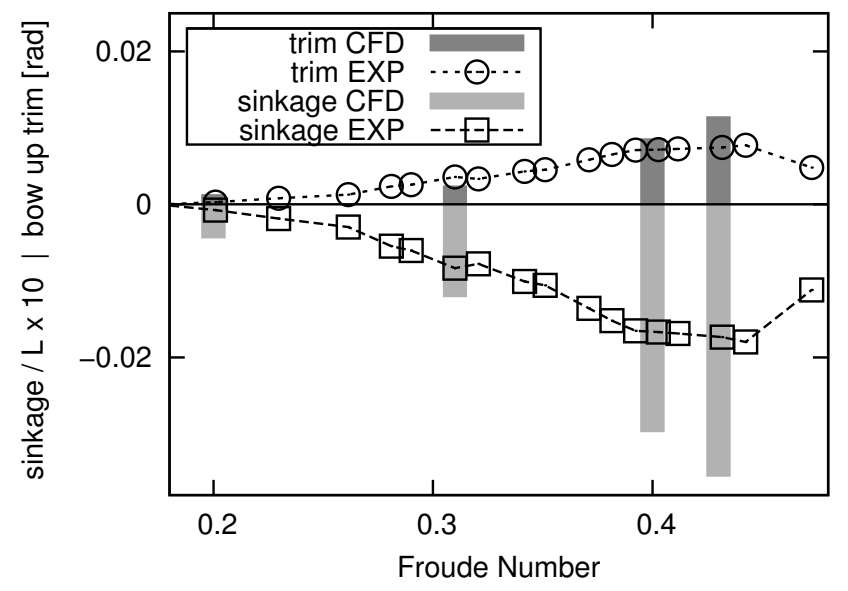

Figure 5: Sinkage and trim for $98 \mathrm{~m}$ high-speed catamaran at 1:22 model scale determined by CFD (shown as vertical bars) and physical towing tank experiments (shown as hollow markers). 


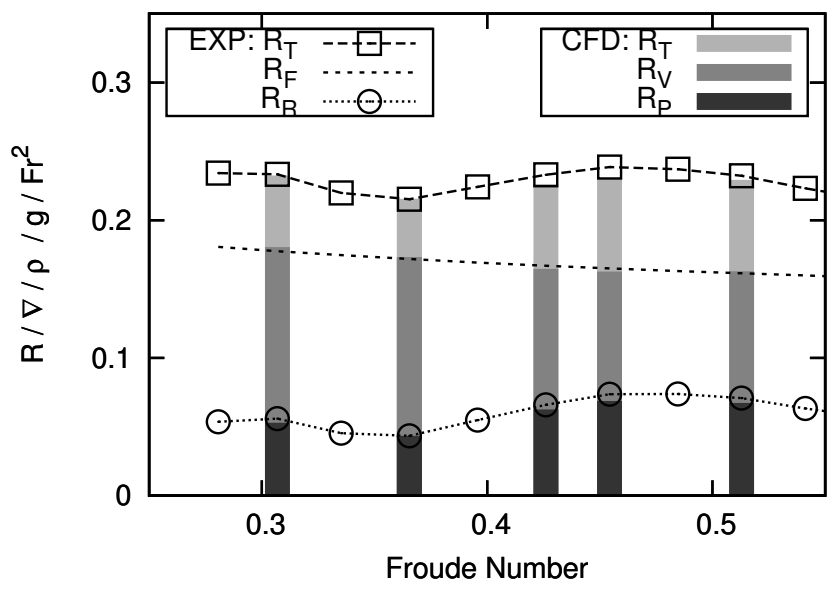

Figure 6: Non-dimensional resistance for $130 \mathrm{~m}$ medium-speed catamaran at 1:50 model scale determined by CFD (shown as vertical bars) and physical towing tank experiments (shown as hollow markers).

\subsection{Model-scale results of $130 \mathrm{~m}$ catamaran}

The calm water resistance for a 1:50 scale model of the $130 \mathrm{~m}$ catamaran was predicted for $\mathrm{Fr}=0.31-0.51$ which corresponds to Reynolds numbers of $\log (R e)=6.5-6.9$. The resulting total drag force is generally lower than that determined in the model test experiments by less than $4 \%$, and the integral of shear stresses deviated by less than 3.5from the ITTC model-ship correlation line. At $F r=0.30$ and 0.45 a hump in the resistance curve can be seen with a hollow at $F r=0.37$. The peak in resistance at $F r=0.31$ is lower than that at $F r=0.45$ and can be associated to wave-making interference, rather than transom drag. Furthermore, the change in dynamic attitude is well predicted by the simulation tool as shown in Figure 7 . 


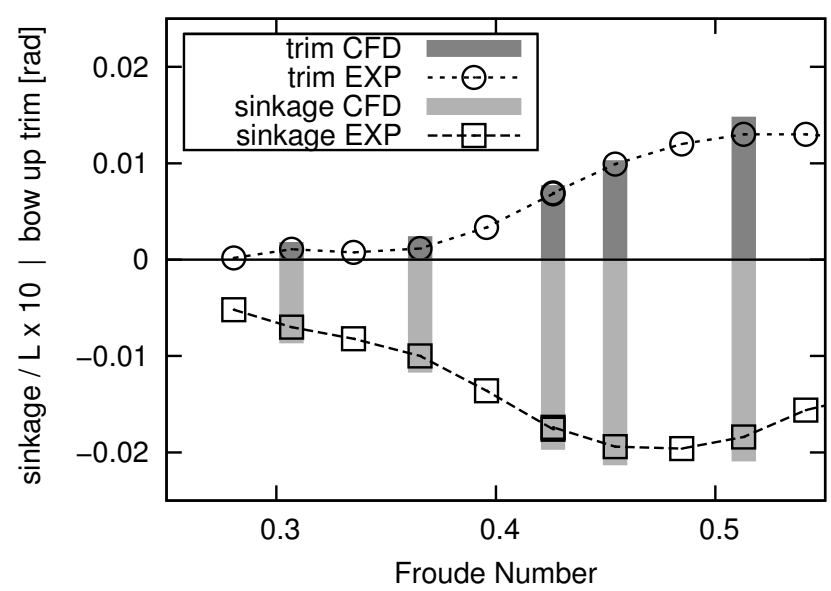

Figure 7: Sinkage and trim for $130 \mathrm{~m}$ medium-speed catamaran at 1:50 model scale determined by CFD (shown as vertical bars) and physical towing tank experiments (shown as hollow markers). 


\section{Verification of near-wall flow}

To assess the changes in shear force coefficient with varying Reynolds number, and the conformity with established model-ship correlation or friction lines, the integral value of shear force coefficient over a two-dimensional flat plate was studied using CFD. The steady state solver simpleFoam of the OpenFOAM CFD toolbox utilising SIMPLE algorithm (semi-implicit pressure-linked equation) was used for this study with standard wall function to model the near wall flow.

The flow over the flat plate was assumed to be two-dimensional to achieve an efficient simulation for a large number of variations and was conducted for $\log (R e)=6.5,7.0,7.5,8.75,9.25$, and 9.75 that correlates to Froude numbers of 0.2 to 0.5 for hull lengths of 2.5 to $200 \mathrm{~m}$. This range therefore encompassed large medium-speed catamarans at both model and full scale. The flow was defined to enter the domain two chord lengths in front of the leading edge of the plate and to leave five chord lengths behind the trailing edge. A free-slip wall was set one chord length opposite from the plate to bound the flow. According to [13] the turbulent boundary layer thickness for unity chord length was estimated to be: $y_{B L}=0.382 R e^{1 / 5}$

Reynolds number was effectively varied by changing kinematic viscosity, while speed and length were set to unity. Within the estimated zone of boundary layer thickness a finite number of cells was distributed with cell expansion ratios of $r_{y}=1.0,1.1,1.2,1.3$ and 1.5. Longitudinally the plate was resolved with 150 cells [14], which was found to be sufficient for a mesh independent solution.

Usually, the dimensionless first cell height is expressed in terms of $y^{+}$, which conveys the distance from the wall based on the boundary layer characteristics. Resolving the boundary layer flow with $y^{+}=1$, where the velocity increases linearly with increasing distance from the wall, would require a relatively small first cell height that results in a very fine boundary layer mesh for flows at high Reynolds numbers. It can be shown that $y_{1}$ scales with $R e^{-0.93}$ which 
leads to a first cell height of 1,000 times finer at $\log (R e)=9.75$ compared to $\log (R e)=6.5$, which would increase the computational resource requirements and may cause numerical issues due to high aspect ratio cells [15]. However, when using wall functions, the first cell height has to reach into the boundary layer where a logarithmic velocity applies. The $y^{+}$of this region increases with increasing Reynolds number and reach an order of magnitude of 2 at modelscale Reynolds number and an order of magnitude of 4 at full-scale Reynolds numbers. Therefore, the first cell height was made dimensionless by geometric means such as the plate length to achieve values that were comparable over a wide range of Reynolds numbers.

The results were compared against other experimentally and numerically derived friction lines, as well as model-ship correlation lines such as that of ITTC, noting that correlation lines express the change in viscous effects rather than the plain friction over a flat plate. Shear force on smooth flat plate The value of numerically determined shear force coefficient $\left(C_{V}\right)$ depends on the geometric properties of the computational grid, such as the first cell height $\left(y_{1}\right)$ and cell expansion ratio $\left(r_{y}=y_{i+1} / y_{i}\right)$ as previously shown by [16]. The present results led to the conclusion that for $r_{y}=1$ a value of $y_{1}$ delivering the highest wall shear stress coefficient $\left(C_{V \max }\right)$ was the most accurate value and was used as a reference value to quantify the deviation in shear force coefficient for varying mesh parameters. Figures $8 \mathrm{a}-8 \mathrm{c}$ show the deviation of shear force coefficient with varying $y_{1}$ values for a range of cell expansion ratios and clearly indicate that both parameters influence the shear force coefficient. For $r_{y}=1, y_{1}$ can be varied over a relatively wide range while still providing higher accuracy than could be ideally achieved with higher $r_{y}$ values. However, if a certain deviation is acceptable, larger $r_{y}$ values for resource sustainable meshes may be chosen. The first cell height providing the highest shear stress was found to be $y_{1} / L \times 10^{3}$ $=1.7,1.0$ and 0.5 for $\log (\operatorname{Re})=6.5,7.5$ and 9.75 , respectively.

This proves that an increase in Reynolds number requires a lower first cell height when using a wall function, but at a considerably lesser extent compared to when a wall function is not used. In addition it was found that the sensitivity 


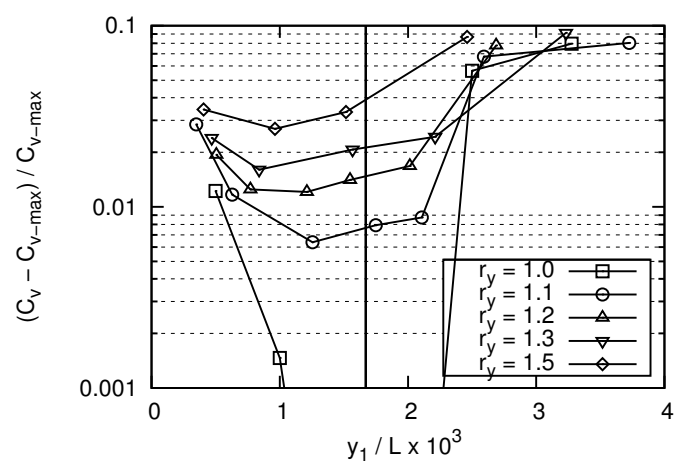

(a) $\log (R e)=6.5$

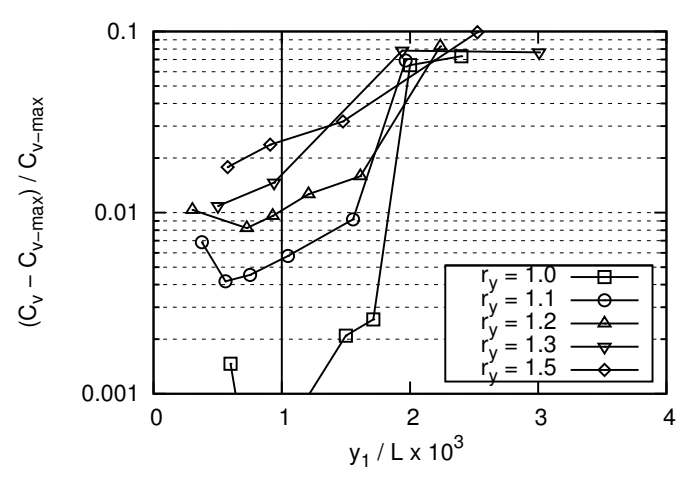

(b) $\log (R e)=7.5$

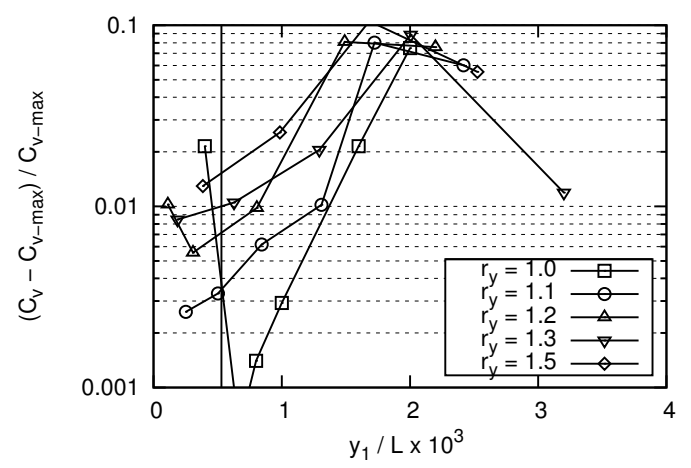

(c) $\log (R e)=9.25$

Figure 8: Effect of varying mesh resolution on shear force coefficient for flow over flat plate at $\log (R e)=6.5,7.5,9.75$ (from top to bottom). Vertical line indicates first cell height for highest achievable shear stress. 


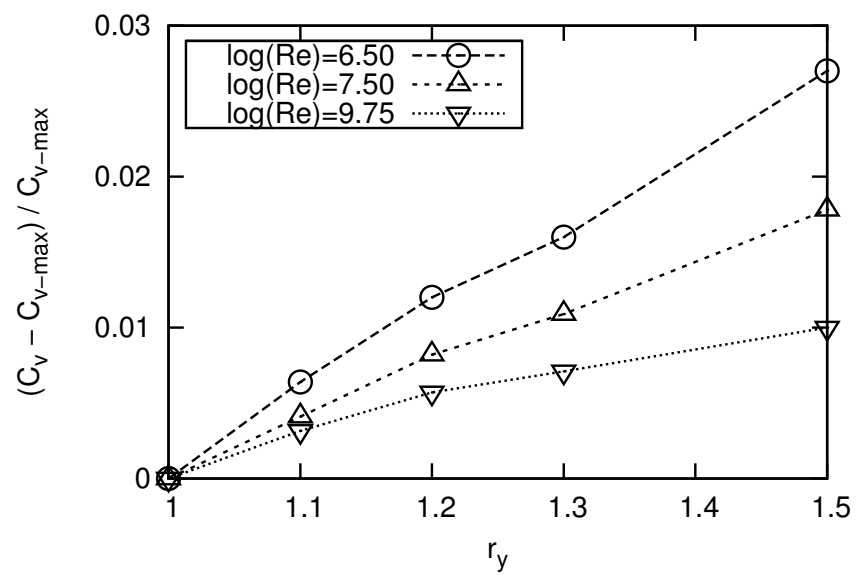

Figure 9: Deviation in shear force coefficient for most appropriate $y_{1}$ for varying cell expansion ratios at different Reynolds numbers.

toward increased ry values decreases for higher Reynolds numbers. Meshes of $y_{1} / L \times 10^{3}=0.51 .0$ with $r_{y} \leq 1.2$ can provide shear force coefficients with less than $2 \%$ deviation from the maximum achievable value for the entire range of Reynolds numbers under consideration. This leads to the conclusion that the same mesh in the proximity of the wall can be used for both model and full scale simulations provided that the first node height is chosen correctly. The relative deviation of shear force with respect to the highest achievable shear force with a certain cell expansion ratio was plotted at different Reynolds numbers (Figure 9 ). The curves show that the deviation at a certain cell expansion ratio is always lower at higher Reynolds numbers and leads to the conclusion that full-scale predictions are less sensitive to variations in near wall mesh properties. Even for relatively large cell expansion ratio of $r_{y}=1.5$ the shear force deviates only $1 \%$ from its most desirable solution. Therefore, at full scale higher cell expansion ratios may be allowed to reduce the cell count compared to simulations at model scale.

Finally, the results of shear force coefficient were compared to established friction and model-ship correlation lines such as those of ITTC and Grigson, 
Katsui, Hughes, and Eca and Hoekstra [16, 17, 18, 19]. The first four lines are

defined as:

$$
\begin{gathered}
C_{F(\text { ITTC })}=\frac{0.075}{(\log (R e)-2)^{2}} \\
C_{F(\text { Hughes })}=\frac{0.067}{(\log (R e)-2)^{2}} \\
C_{F(\text { Grigson })}=f_{G} \times C_{F(I T T C)} \\
f_{1-6}=\left\{\begin{array}{l}
G_{1}+G_{2}\left(\log (R e)-F_{1}\right)^{2}-G_{3}\left(\log (R e)-F_{1}\right)^{4}, \text { for } 6 \leq \log (R e) \leq 7.3 \\
G_{4}+G_{5}\left(\log (R e)-F_{2}\right)-G_{6}\left(\log (R e)-F_{2}\right)^{2}, \text { for } 7.3 \leq \log (R e) \leq 9
\end{array}\right. \\
C_{F(\text { Katsui })}=\frac{0.0066577}{\log (R e)-4.3762)^{\alpha}} \\
\alpha=\log (\operatorname{Re})+0.56725
\end{gathered}
$$

The line of Eca and Hoekstra [16] was derived using CFD without using wall functions. The lines are compared in Figure 10 where good agreement between the current method and the results of Eca and Hoekstra were found over the whole range of Reynolds numbers. At model scale agreement with Hughes line was observed and at full-scale with the lines of ITTC, Grigson and Katsui.

\subsection{Shear force on rough flat plate}

A full-scale ship hull cannot be assumed to be hydraulically smooth, as its surface contains roughness due to paint, marine growth, plate dents and weld seams $[20,21]$. In the numerical model the effect of surface roughness on the boundary layer flow was implemented [22] and applied to the flat plate. Roughness values of $k_{S} / L=1 \times 10^{-6}$ (SR1) and $2 \times 10^{-6}$ (SR2) were chosen which correspond to a $100 \mu \mathrm{m}$ and $200 \mu \mathrm{m}$ sand grain roughness on $100 \mathrm{~m}$ long ship hull. In the range of full-scale Reynolds number $(8<\log (R e)<10)$ prediction for the shear force coefficient was made using CFD, ITTC (7.5-02-0301.4) and an empirical estimate of Schlichting [23]. The latter assumes a fully 


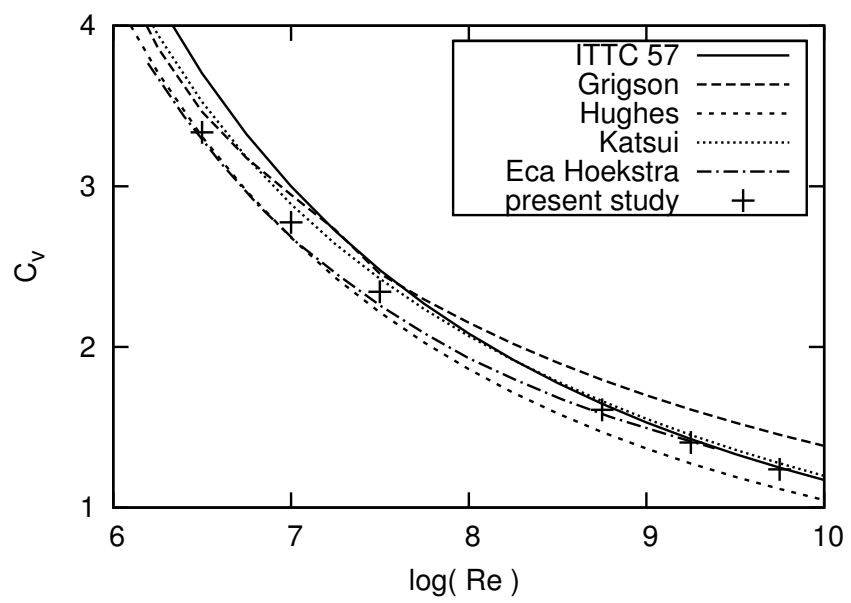

Figure 10: Present results for shear force coefficient compared to established correlation lines for model-ship extrapolation over a wide range of Reynolds numbers.

rough wall for which the local friction coefficient solely depends on the surface roughness and not on the Reynolds number:

$$
C_{f}=\left(1.89-1.62 \log \left(k_{S} / L\right)\right)-2.5
$$

Figure 11 shows that for CFD predictions at $\log (R e)=8.25-9.25$ the shear force coefficient increases with increasing Reynolds number. It branches of from the curve of the shear force coefficient for a smooth flat plate and increases towards the value determined by Schlichting for a fully rough plate which corresponds to literature [13]. For $\log (R e) \leq 9.25$ predictions by CFD and Schlichting agree well and therefore it is assumed that the predictions are physically adequate. Contrary, the ITTC prediction for a rough surface monotonically decreases with increasing Reynolds number over the whole range of consideration. However, at $\log (R e)=8.75$ the predictions by ITTC and CFD are of comparable magnitude, whereas at lower Reynolds numbers ITTC predicts a larger impact of roughness on the drag force lower impact at higher Reynolds numbers. The relative increase in shear force for a relative roughness of $k_{S} / L=1 \times 10^{-6}(\mathrm{SR} 1)$ 


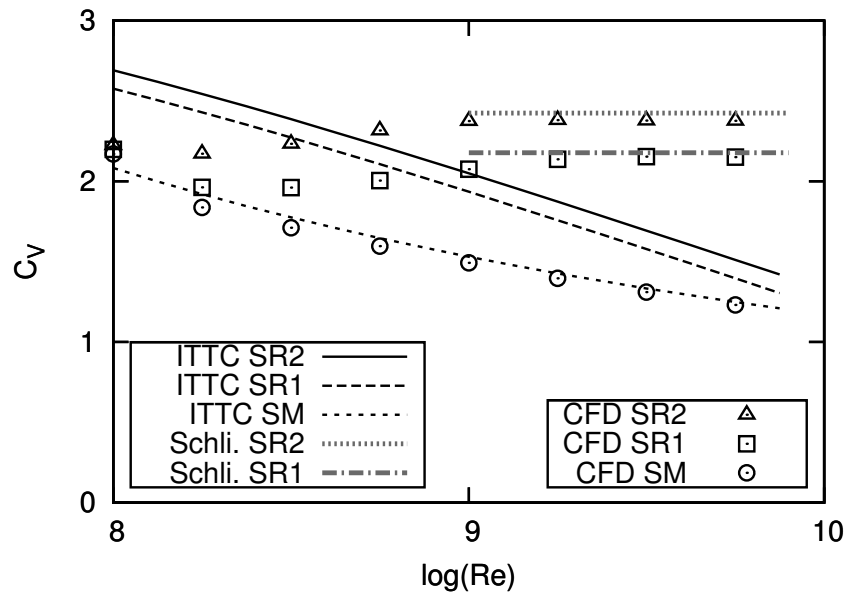

Figure 11: Integral values of shear force coefficient $\left(C_{V}\right)$ for the flow over a flat plate being smooth $(\mathrm{SM})$ and rough: $k_{S} / L=1 \times 10^{-6}$ (SR1) and $2 \times 10^{-6}$ (SR2). Results were obtained by CFD, ITTC (7.5-02-03-01.4) and empirically by estimating local skin friction by Schlichting (Schli.) for fully rough walls.

and $2 \times 10^{-6}$ (SR2) referring to equivalent sand grain roughness with respect to a smooth plate can be seen in Figure 12. This highlights the contrary trend of the shear force coefficient between numerical prediction and empirical estimate of ITTC for Reynolds numbers exceeding $\log (R e)>9$. Furthermore the range of Reynolds numbers for a $98 \mathrm{~m}$ and a $130 \mathrm{~m}$ at medium-speed Froude numbers of $F r=0.35-0.45$ were plotted to illustrate that the adverse effect of surface roughness is expected to be more pronounced at larger vessels or higher speeds. At $\log (R e)=9.25$ the values from CFD and Schlichting is larger by $18 \%$ and $27 \%$ for a roughness of $k_{S} / L=1$ and $2 \times 10^{-6}$, respectively. This emphasises that the ITTC value may either be only valid for a small range of Reynolds numbers or is already including a correlation allowance factor. 


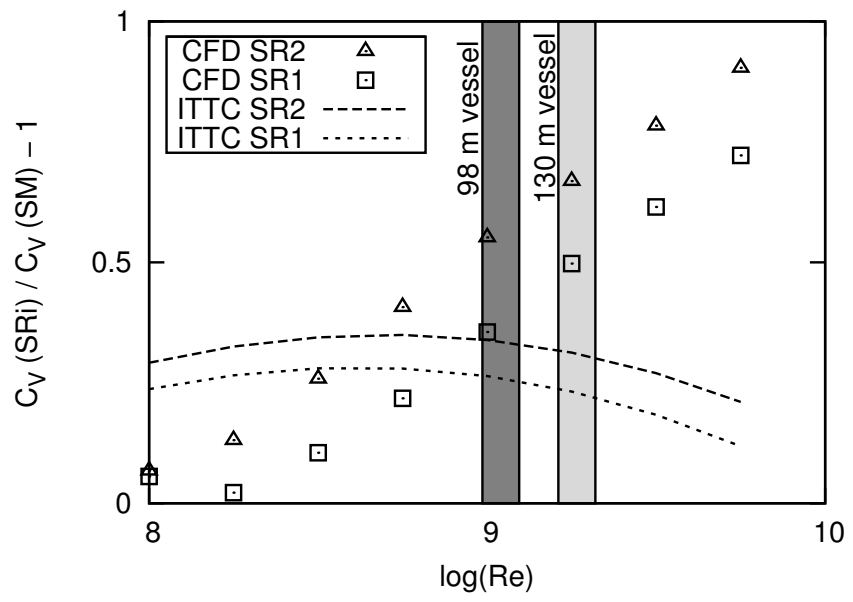

Figure 12: Relative increase of shear force coefficient compared to hydraulically smooth plate for equivalent sand grain roughness of $k_{S} / L=1 \times 10^{-6}$ (SR1) and $2 \times 10^{-6}$ (SR2). Range of Reynolds number for a $98 \mathrm{~m}$ and a $130 \mathrm{~m}$ vessel at medium speeds $(F r=0.35-0.45)$ are indicated. 


\section{Validation of full-scale drag}

Sea trial data and extrapolated model test data was utilised to validate the full-scale CFD predictions. Full-scale speeds of 12, 18, 23.5 and 25 knots for the $98 \mathrm{~m}$ catamaran were considered which correspond to the model scale results at

$345 \mathrm{Fr}=0.20,0.31,0.40$ and 0.43 . The drag for the $130 \mathrm{~m}$ catamaran was predicted at hollow and hump conditions $F r=0.37$ and 0.45 which equals 25 and 30 knots for the full scale vessel. Reynolds numbers range from $\log (R e)=8.8-9.3$ when considering both cases.

\subsection{Drag force from full-scale sea trials}

During full-scale sea trials of a $98 \mathrm{~m}$ high-speed catamaran [24] the shaft power and associated speed were measured in deep water conditions $(h / L>$ 10) at wind speeds below 25 knots and waves of $5 \mathrm{ft}$ at a $7 \mathrm{~s}$ period. The shaft power was obtained by averaging the values from $2-3$ runs per powering condition with measurement uncertainty reported to be below $2 \%$. Referring to the thrust curves of the waterjet propulsors, which were made available by the manufacturer, the measured delivered power for a certain speed could be associated to a nominal thrust per waterjet unit. The obtained thrust value was considered as being equal to the resistance as it was assumed that both the thrust deduction and wake fraction were negligible, as proposed by Iliopulos et al. [9].

\subsection{Extrapolation of model test data}

\subsubsection{Extrapolation for deep water}

The measurements from the physical model test were extrapolated using the ITTC guidelines for conventional vessels (7.5-02-02-01.4) and high-speed marine vessels (HSMV) (7.5-02-05-01). The difference between the two methods is in their respective approaches to using a form factor, which is not recommended when utilising guidelines for HSMV due to the flow past a deep, dry transom and hence $(1+k)$ is set as 1.0. The total resistance coefficient was calculated 
by:

$$
C_{T s}=C_{T m}\left((1+k) \times\left(C_{F m} C_{F s}\right)+d C_{F}+C_{A}+C_{A A S}\right.
$$

where $C_{T m}$ is the total resistance coefficient at model scale, $C_{F}$ is the frictional resistance according to the model-ship correlation line of ITTC (7.5-02-02-01), $d C_{F}$ the roughness allowance, $C_{A}$ the correlation allowance and $C_{A A S}$ the air resistance coefficient. The latter coefficients can be determined as:

$$
\begin{gathered}
d C_{F}=0.044\left(\left(k_{S} / L\right)^{1 / 3}-10 R e^{-1 / 3}\right)+0.000125 \\
C_{A}=(5.68-0.6 \log (\text { Re })) \times 10^{-3} \\
C_{A A S}=C_{D} A \times \rho_{\text {air }} / \rho_{\text {water }} \times A_{\text {proj }} / S_{W}
\end{gathered}
$$

where $k_{S}$ is the surface roughness which is assumed to be 100 and $200 \mu \mathrm{m}, L$ the ship length, $C_{D A}$ the drag coefficient of the superstructure which was assumed to be $C_{D A}=0.446$ for a typical INCAT high-speed catamarans [25] and a projected area of the superstructure of $A_{p r o j}=0.04 L^{2} . S_{W}$ denotes the wetted surface area. As an alternative the Grigson line [17] was utilised for model ship correlation, but the value of extrapolated resistance was between that of the two methods presented above, differing by $2-3 \%$ for the speeds under consideration whereas a form factor higher than unity leads to lower results.

\subsubsection{Finite Depth Corrections}

For the model tests of the $98 \mathrm{~m}$ vessel the depth Froude number for the current case was relatively high $\left(F r_{d}<0.75\right)$ and ITTC recommended procedures (7.5-02-02-01.4) were consulted for a correction of the model test experiments. The approach of Schuster [26] was considered to correct the model test data for finite water effects such as blockage and increased wave-making to make the test data recorded in finite water applicable for unbounded water cases. The method considers the relative change of effective velocity $(d u / U)$ around the ship model, such as:

$$
C_{T}=R_{T} /\left(\rho / 2 U^{2}(1+d u / U)^{2} S_{W}\right)
$$


where $d u / U$ is given by

$$
d U / U=m /\left(1-m-F r_{h}^{2}\right)+\left(1-R_{F} / R_{T}\right) 2 / 3 F r_{h}^{10}
$$

with $m=A_{x} /(b \times h)$. This formula predicts an increase in residuary resistance of $3 \%$ at $F r=0.31$ and $15 \%$ at $F r=0.43$ due to finite water effects. Since the finite water effects appear not to be negligible for the current case, this approach was utilised for extrapolation of the model-test data of the $98 \mathrm{~m}$ highspeed catamaran.

\subsection{Full-scale CFD results}

The full-scale resistance from the CFD data was obtained by repeating the simulations using model scale dimensions at full-scale Reynolds numbers, with an extended domain to ensure that finite water effects did not significantly affect the results. The depth of the domain was extended to $h / L=1.0$ in accordance with ITTC guidelines (7.5-03-02-03) and surface roughness was taken into account in the numerical model [22]. Since the original surface roughness of the full-scale vessel at the time of operation was unknown exactly, the values of $k_{S}$ ${ }_{385}=100$ and $200 \mu \mathrm{m}$ were used. Whilst according to ITTC recommended procedures (7.5-02-02-01.4), $150 \mu \mathrm{m}$ should be chosen if the exact value is not known, using two values allows an estimate of varied surface roughness on the effective power, as the boundary layer around the ship is influenced by hull imperfections that add to the initial surface roughness, such as due to weld lines, plate dents, difference in plate thickness and marine growth.

A correction for air drag was added in an identical approach to that for the extrapolated model test data. This resistance component could also have been determined using CFD, but that was considered as being beyond the scope of this work. Furthermore, the air drag was neither subject to laminar-turbulent transition effects nor Froude number dependent scaling and therefore the current approach is considered as being appropriate. 


\subsection{Comparison of full-scale drag for $98 \mathrm{~m}$ catamaran}

A detailed comparison of resistance from the different prediction methods including 100 and $200 \mu \mathrm{m}$ surface roughness can be seen in Figure 13. The resistance was subdivided as specified in section 3.2 with additionally taking into account the drag due to surface roughness (RdCF (ITTC)), correlation allowance (RA (ITTC)) and wind drag due to the superstructure (RAA (ITTC)), as proposed by ITTC (7.5-02-02-01.4). In the numerical simulation the drag due to surface roughness ( $\mathrm{RdCF}(\mathrm{CFD})$ ) was determined by the difference in drag due to tangential stresses for cases with and without surface roughness included, as it cannot be separately isolated.

For speeds at $F r=0.20$ and 0.31 the CFD prediction was below the result obtained from power measurements of sea trials by $20-23 \%$ and $5-10 \%$ depending on the surface roughness applied. For $F r=0.40$ and 0.43 the drag obtained from CFD deviated by $1-5 \%$ and $-3-3 \%$, respectively. For the latter two speeds the extrapolated model test data was within the numerically predicted values. Numerical differences of the different prediction methods are presented in Table 1. The deviations are relative to the value obtained from the sea trial measurements and expressed as:

The differences in resistance components are summarised in Table 2. For Fr $=0.20$ the differences between pressure and residuary drag are between $49 \%$ and $104 \%$, but the shear force over a smooth hull is only $1 \%$ larger in CFD. When considering surface roughness the relative difference increases to $10 \%$ and $23 \%$ for 100 and $200 \mu \mathrm{m}$ of equivalent sand grain roughness. For speeds from $F r=0.31$ to 0.43 the maximum deviation of pressure resistance when compared to residuary resistance is less than $8 \%$ and $11-23 \%$ when using a form factor. If the latter is used the viscous drag force in CFD is under-estimated by $19 \%$ whereas this difference decreases to below $5 \%$ when no form factor was used for a smooth hull. When including surface roughness, CFD delivers results that are less than $4 \%$ higher than ITTC predictions for $100 \mu \mathrm{m}$ and $15-16 \%$ higher when $200 \mu \mathrm{m}$ of equivalent sand grain roughness is assumed. 


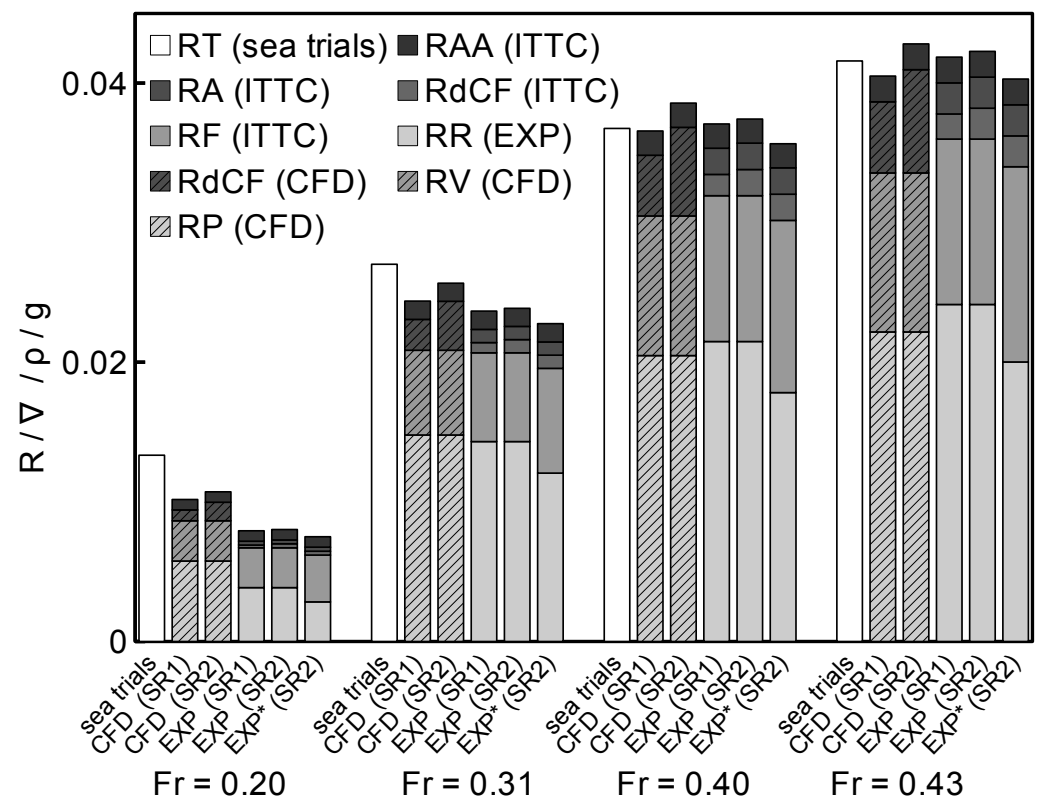

Figure 13: Full-scale drag predictions for $98 \mathrm{~m}$ medium-speed catamaran from model test experiments (EXP) (with and without form factor, ${ }^{*}$ indicates usage of form factor of $(1+k)=1.18)$ and CFD (CFD) at $F r=0.20-0.43$ at two levels of surface roughness. SR1: $k_{S}=100 \mu \mathrm{m}, \mathrm{SR} 2: k_{S}=200 \mu \mathrm{m}$. 
Table 1: Relative deviation of predicted drag for $98 \mathrm{~m}$ catamaran using CFD and extrapolated model test data corrected for shallow water by the approach of Schuster [26] with and without form factor with respect to drag derived from full-scale powering measurements. $k_{S}$ of $100 \mu \mathrm{m}$ and $200 \mu \mathrm{m}$ was considered.

\begin{tabular}{|c|c|c|c|c|c|}
\hline \multirow[t]{2}{*}{$\begin{array}{l}\text { Speed } \\
{[k n]}\end{array}$} & \multicolumn{5}{|c|}{$\begin{array}{l}\text { Relative deviation of total resisitance } \\
\text { CFD prediction and extrapolated model tests } \\
\text { vs results from sea trials measurements }\end{array}$} \\
\hline & \multicolumn{2}{|c|}{$\mathrm{CFD}$} & \multicolumn{3}{|c|}{ ITTC extrapolated model test } \\
\hline$(1+k)$ & \multicolumn{4}{|c|}{1.0} & 1.18 \\
\hline$k_{S}$ & $100 \mu \mathrm{m}$ & $200 \mu \mathrm{m}$ & $100 \mu \mathrm{m}$ & \multicolumn{2}{|c|}{$200 \mu \mathrm{m}$} \\
\hline 12 & -0.234 & -0.197 & -0.406 & -0.399 & -0.438 \\
\hline 18 & -0.098 & -0.050 & -0.125 & -0.117 & -0.158 \\
\hline 23.5 & 0.005 & 0.049 & 0.009 & 0.018 & -0.030 \\
\hline 25 & -0.026 & 0.030 & 0.007 & 0.017 & -0.031 \\
\hline
\end{tabular}

Table 2: Relative deviation of resistance components of $98 \mathrm{~m}$ catamaran determined by CFD and extrapolated model test experiment for a smooth hull. Positive values indicate that CFD prediction exceeds extrapolated quantity.

\begin{tabular}{|c|c|c|c|c|c|c|}
\hline $\begin{array}{l}\text { Froude } \\
\text { number }\end{array}$ & \multicolumn{6}{|c|}{$\begin{array}{l}\text { Relative deviation of resistance components } \\
\qquad \text { CFD vs extrapolated model test results }\end{array}$} \\
\hline & \multicolumn{2}{|c|}{$\mathrm{RP} / \mathrm{RR}-1$} & \multicolumn{4}{|c|}{$\mathrm{RV} / \mathrm{RF}-1$} \\
\hline$(1+k)$ & 1.0 & & .18 & \multicolumn{3}{|c|}{1.0} \\
\hline$k_{S}$ & \multicolumn{4}{|c|}{ smooth } & $100 \mu \mathrm{m}$ & $200 \mu \mathrm{m}$ \\
\hline 0.20 & 0.49 & 1.04 & -0.15 & 0.01 & 0.10 & 0.23 \\
\hline 0.31 & 0.03 & 0.23 & -0.19 & -0.05 & 0.03 & 0.16 \\
\hline 0.40 & -0.05 & 0.15 & -0.19 & -0.04 & 0.04 & 0.15 \\
\hline 0.43 & -0.08 & 0.11 & -0.19 & -0.04 & 0.04 & 0.15 \\
\hline
\end{tabular}




\subsection{Comparison of full-scale drag for $130 \mathrm{~m}$ catamaran}

The numerical predictions were compared to the extrapolated model test data following ITTC recommendations with a form factor $((1+k)=1.1)$ and

without, and a smooth surface as well as surface roughness of 100 and $200 \mu \mathrm{m}$ of equivalent sand grain roughness was included.

At $F r=0.37$ the CFD-predicted resistance for a smooth hull was $5-13 \%$ larger than extrapolated model test results. Depending if a form factor was used or not. The discrepancy increased to $17-27 \%$ if a surface roughness of 100 and $200 \mu \mathrm{m}$ was taken into account. At $F r=0.45$ the difference between the CFD prediction and extrapolated model test data was below $5 \%$, but reached values of $11-21 \%$ with surface roughness included in the prediction method.

Figure 3 shows the agreement between the CFD predictions and extrapolated model test results including a subdivision into resistance components and

${ }_{440}$ Table 3 shows a quantitative comparison of total resistance. Table 4 quantifies the relative difference in pressure related $(\mathrm{RP})$ and residuary $(\mathrm{RR})$ resistance when using form factor and when not for both speeds. Furthermore, it shows the relative differences in viscous resistance (RV) with respect to frictional resistance (RF) for a smooth hull and also with included roughness and correlation allowance as obtained by ITTC (7.5-02-02-01.4). Residuary resistance deviates up to $57 \%$ and $13 \%$, while frictional resistance deviates below $11 \%$ and $5 \%$ when using and not using a form factor. For $(1+\mathrm{k})=1.0$ the frictional resistance including roughness and correlation allowance is $44 \%-56 \%$ higher in CFD prediction than it is compared to empirically determined values when following recommendations by ITTC (7.5-02-02-01.4). 


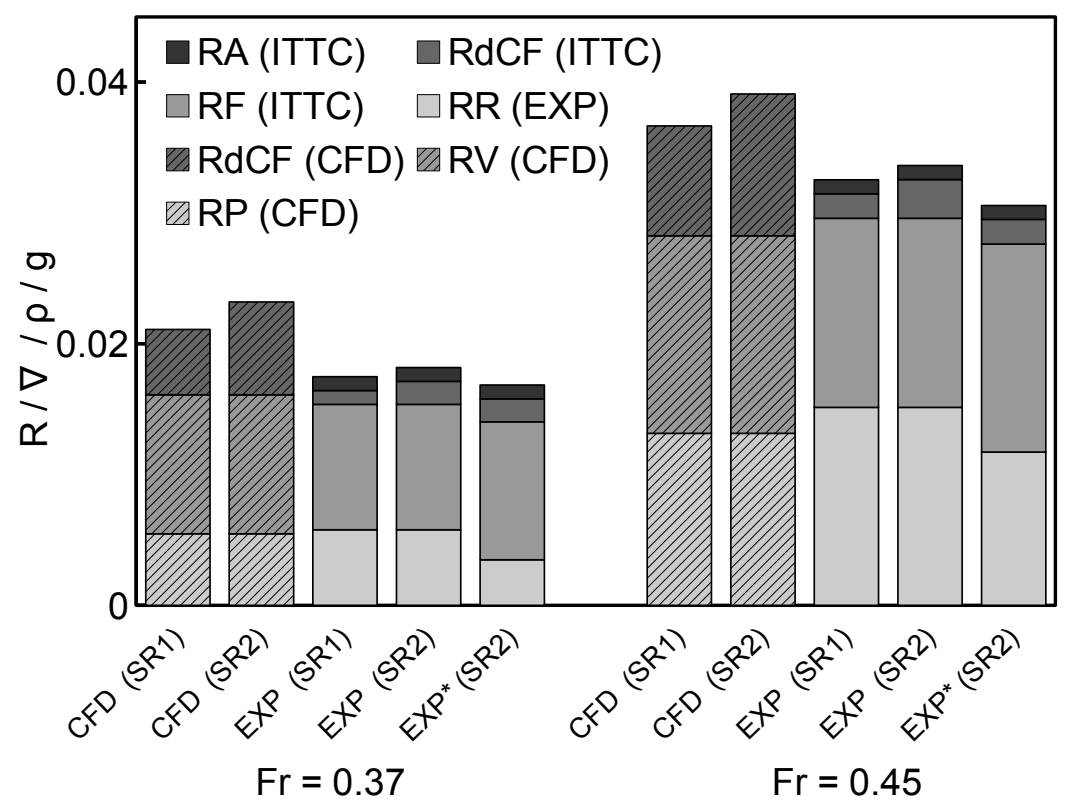

Figure 14: Full scale drag predictions for $130 \mathrm{~m}$ catamaran from model test experiments (EXP) (with and without form factor, ${ }^{*}$ indicates $\left.(1+k)=1.10\right)$ and CFD (CFD) of bare hull with no superstructure of $130 \mathrm{~m}$ medium-speed catamaran at $F r=0.37$ and 0.45 at two levels of surface roughness. SR1: $k_{S}=100 \mu \mathrm{m}, \mathrm{SR} 2: k_{S}=200 \mu \mathrm{m}$. 
Table 3: Relative deviation of resistance components of $130 \mathrm{~m}$ catamaran determined by CFD and extrapolated model test experiment. Positive values indicate that CFD prediction exceeds extrapolated quantity.

\begin{tabular}{|c|c|c|c|c|c|}
\hline $\begin{array}{l}\text { Froude } \\
\text { number }\end{array}$ & \multicolumn{5}{|c|}{$\begin{array}{l}\text { Relative deviation of total resistance } \\
\text { CFD vs. and extrapolated model test results }\end{array}$} \\
\hline & \multicolumn{2}{|c|}{$\mathrm{RP} / \mathrm{RR}-1$} & \multicolumn{3}{|c|}{$\mathrm{RV} / \mathrm{RF}-1$} \\
\hline$(1+k)$ & 1.0 & 1.1 & & & 1.1 \\
\hline$k_{S}$ & \multicolumn{2}{|c|}{$0 \mu \mathrm{m}$, no $C_{A}$} & $100 \mu \mathrm{m}$ & \multicolumn{2}{|c|}{$200 \mu \mathrm{m}$} \\
\hline 0.37 & -0.045 & -0.128 & -0.171 & -0.212 & -0.274 \\
\hline 0.45 & 0.047 & -0.022 & -0.113 & -0.140 & -0.218 \\
\hline
\end{tabular}

Table 4: Relative deviation of resistance components determined by CFD and extrapolated model test experiment. Positive values indicate that CFD prediction exceeds extrapolated quantity.

\begin{tabular}{|c|c|c|c|c|c|c|}
\hline $\begin{array}{l}\text { Froude } \\
\text { number }\end{array}$ & \multicolumn{6}{|c|}{$\begin{array}{l}\text { Relative deviation of resistance components: } \\
\qquad \text { CFD vs extrapolated model test results }\end{array}$} \\
\hline & \multicolumn{2}{|c|}{ RP/RR-1 } & \multicolumn{4}{|c|}{$\mathrm{RV} / \mathrm{RF} \quad 1$} \\
\hline$(1+k)$ & 1.0 & \multicolumn{2}{|c|}{1.18} & \multicolumn{3}{|c|}{1.0} \\
\hline$k_{S}$ & \multicolumn{2}{|c|}{$0 \mu \mathrm{m}$} & \multicolumn{2}{|c|}{$0 \mu \mathrm{m}$} & $100 \mu \mathrm{m}$ & $200 \mu \mathrm{m}$ \\
\hline 0.37 & -0.05 & 0.57 & 0.11 & 0.01 & 0.47 & 0.56 \\
\hline 0.45 & -0.13 & 0.12 & 0.04 & -0.05 & 0.44 & 0.49 \\
\hline
\end{tabular}




\section{Discussion}

\subsection{Full-scale drag from sea trials}

A CFD-based approach, which uses verification with model test experiments, to determine the full-scale resistance of a surface vessel was presented in section 2. The results from using this approach for a $98 \mathrm{~m}$ catamaran were validated using resistance values obtained from power measurements at full-scale sea trials. All three approaches (CFD simulation, model test extrapolation and sea trial estimates) showed a deviation below $5 \%$ for $F r=0.40,0.43$. However, the resistance from the sea trials was larger at $F r=0.20,0.31$ by up to $44 \%$. To the authors knowledge the effect of thrust deduction can be considered as being negligible for $\mathrm{Fr}>0.4$ [5], but wake fraction will have values around $w=0.1$, which is in accordance with estimates by Bulten [27] and de Cock [28]. For speeds of $\mathrm{Fr}<0.4$ values of thrust deduction may tend towards unity [5] with decreasing speed while wake fraction remains unchanged. Taking wake

$$
45 \text { fraction and thrust deduction into account resistance can be determined by: }
$$$$
R_{T}=(1-t) /(1-w) \times T
$$

Hence the resistance when utilising the proposed method will be under estimated by $10 \%$ for $w=0.1$ and $t=0.0$ and over estimated by $45 \%$ for $w=0.1$ and $t=0.5$. However, given the agreement for $F r>0.4$ between the result from sea trials when neglecting wake fraction and thrust deduction and the CFD fullscale prediction and model test data extrapolation, it can be assumed that the effect of thrust deduction and wake fraction is counteracted by environmental influences such as wind and waves, marine growth on the hull or mechanical losses resulting from assemblage of the propulsion plant.

Iliopulos et al. [9] state that neglecting wake fraction and thrust deduction to obtain resistance from thrust effectively is the inverse procedure of choosing a waterjet size for a given resistance. Therefore, this method has to be considered as being practical rather than physically accurate. 


\subsection{Correlation allowance}

The results of the $130 \mathrm{~m}$ catamaran show that the additional resistance due to a rough surface exceeds the correlation allowance estimated by ITTC (7.502-03-01.4). For large vessels recommended correlation allowances including roughness allowance can smaller than zero [3]. This originates from the correlation of full-scale sea trials and model test experiments of conventional ships, but it is physically unreasonable that including surface roughness reduces the total drag. A possible reason could be that using the ITTC model-ship correlation line over-estimates the viscous and residuary resistance components at such large Reynolds numbers $(\log (R e)>9.0)$ which needs to be compensated. However, considering the results in section 4.1, 4.2 and 5.5 this would imply that near-wall flows for smooth and rough surfaces are incorrectly predicted by CFD and empirical approaches under consideration. Another possibility is that the propulsion plant may be working more efficiently at such large Reynolds numbers due to reduced viscous losses. Therefore, a total resistance below the physically accurate drag force can be considered for choosing an appropriate engine size. Further research is required to numerically quantify added resistance due to surface roughness when Reynolds number exceeds $\log (R e)>9.0$.

\subsection{Verification of CFD simulations}

The verification of the CFD set up was necessary to assure that the main flow features were sufficiently accurately resolved. For the current vessels these features may include the wave-making, the demihull interaction, and the flow separation at the transom stern. Therefore, the successful verification allows to use the specified spatial resolution for accurately predicting the full-scale resistance of similar ships that provide comparable geometric and hydrodynamic features. Based on the current example the authors would claim legitimacy of the set up for reasonable changes in slenderness, transom immersion, or draft. However, if the vessel shape will be considerably different, such as being a monohull vessel or having features including a pronounced fore shoulder or strongly convex buttock or water lines in the aft section a reverification of the CFD set up 
may be required. A geometrically identical or at least similar hull form should 510 be used to assure that results for the full-scale drag are physically meaningful. 


\section{Conclusions}

A novel CFD-based methodology was developed and it was shown that it is capable of accurately predicting the full-scale resistance of a marine surface ship in conjunction with model test experiments. Hence it can be interpreted as a numerical tool for model test result extrapolation. The predictions were successfully validated for large medium-speed catamarans by comparison with results obtained from full-scale measurements.

The CFD simulations were found to be capable of precisely estimating the drag of medium-speed catamarans at 1:50 and 1:22 model scale, with deviations in resistance being below $8 \%$. Shear force was found to be in accordance with several friction and model-ship correlation lines for both model-scale and fullscale Reynolds numbers and it was shown that an identical near wall resolution can be used throughout the entire range of Reynolds numbers under consideration $(6.5<\log (R e)<9.75)$. Full-scale Reynolds numbers were achieved in the simulation by altering viscosity and keeping the linear dimensions at model scale, which allows the use of the same meshes at both model-scale verification and full-scale prediction. Furthermore, the effect of surface roughness was included and it was found that for $\log (R e)>8.75$ the impact of roughness on the total drag is more pronounced in CFD simulations when compared to the empirical suggestion from ITTC, while CFD predictions for fully rough conditions agreed with values found in literature on flow physics.

A validation of the full-scale resistance obtained by CFD simulations was conducted using resistance force derived from measured shaft power and extrapolated model test data. For $F r \geq 0.40$ deviation of the CFD result was below $5 \%$ from the full-scale reference values; the same agreement was achieved for the different model test extrapolations. For $F r \leq 0.31$ the CFD prediction was between $5-23 \%$ below the sea trial estimate while model test extrapolation was $12-44 \%$ below values estimated from sea trials. When considering the 130 m catamaran for $F r=0.37$ the CFD prediction was $17-27 \%$ larger than extrapolated model test results, depending on extrapolation method and surface 
roughness and $11-21 \%$ larger for $F r=0.45$. Full-scale resistance considering a smooth hull was $5-13 \%$ higher when predicted by CFD at $F r=0.37$, while at $F r=0.45$ smooth hull resistance varied only from $-5-2 \%$. Small changes in vessel geometry may be allowed without verifying the CFD set up all over again, which makes this a powerful tool for hull form studies at hydrodynamic full scale. However, when significant changes to the ship hull shape are applied, the CFD set up may require to be verified with an applicable model test comparison to assure the delivery of accurate results.

This study underlined the versatility of computational fluid dynamics in the ship design process and its applicability for full-scale simulations including accurate resistance predictions that take surface roughness into account. Furthermore it highlights the importance of, but also the difficulty to, applying an appropriate roughness and correlation allowance to large medium-speed catamarans, especially when Reynolds numbers reach such high values. 


\section{Future work}

Shallow water effects can significantly influence the performance of surface vessels, especially at medium speed Froude numbers, and the ITTC guidelines are not so well established for those critical Froude numbers. The applicability this novel CFD approach for shallow water cases should be investigated for medium-speed catamarans.

Furthermore, a deviation between CFD and model test predictions at speeds below $\mathrm{Fr}<0.31$ was observed. At these speeds the transom is undergoing the change from fully wet to fully dry. This aeration process may not be reliably resolved with the current computational resolution. A study focussing on the flow around the partially wetted transom should be undertaken to quantify difference that occur in numerical simulations and model test experiments.

Finally, the correlation allowance at Reynolds numbers exceeding $\log (R e)>$ 9.0 needs to be subject to further investigation. Sea trial measurements and detailed analysis of the propulsion plant should be undertaken to probe this phenomenon. 


\section{Acknowledgements}

This research has been conducted as part of a collaborative research project between INCAT, Revolution Design, MARIN, Wärtsilä and the Australian Maritime College at the University of Tasmania. It was supported under Australian Research Council's Linkage Projects funding scheme (project number LP110100080).

Furthermore, the authors would like to express their gratitude to Stuart Friezer for his input to and support of this research as well as Prof. Michael R. Davis for reviewing this article prior to submission. 
[1] G. Davidson, T. R. Roberts, S. Friezer, M. R. Davis, N. Bose, G. Thomas, J. Binns, R. Verbeek, Maximising Efficiency and Minimising Cost in High Speed Craft, in: International Conference on Fast Sea Transportation, Vol. 11, 2011.

[2] M. Haase, G. Davidson, S. Friezer, J. Binns, G. Thomas, N. Bose, On the macro hydrodynamic design of highly efficient medium-speed catamarans with minimum resistance, International Journal of Maritime Engineering.

[3] V. Bertram, Practical Ship Hydrodynamics, Butterworth-Heinemann, 2000.

${ }_{590}^{5}$ [4] M. Haase, G. Davidson, S. Friezer, J. Binns, G. Thomas, N. Bose, Hydrodynamic hull form design space exploration of large medium-speed catamarans using full-scale CFD, International Journal of Maritime Engineering in Press.

[5] F. H. Lafeber, R. Hulshof, J. H. Allema, J. H. de Jong, Calm Water Test for the JHSV Wave Piercing Catamaran Final Report, Tech. rep., MARIN (2008).

[6] H. C. Raven, A. van der Ploeg, A. R. Starke, L. Eca, Towards a CFD-based prediction of ship performance - Progress in predicting full-scale resistance and scale effects, in: Proceedings of RINA International Conference Marine CFD, 2008.

[7] K. Hochkirch, B. Mallol, On the Importance of Full-Scale CFD Simulations for Ships, in: International Conference on Computer Applications and Information Technology in Maritime Industries, 2013.

[8] M. Haase, F. Iliopulos, G. Davidson, S. Friezer, G. Thomas, J. Binns, N. Bose, J. Lavroff, M. R. Davis, Application of RANSE based simulations for resistance prediction of medium-speed catamarans at different scales, in: Proceedings of 18th Australasian Fluid Mechanics Conference, 2012. 
[9] F. Iliopulos, J. Lavroff, M. R. Davis, J. Binns, G. Davidson, R. Verbeek, Validation for full scale Ranse simulation of resistance prediction for high speed catamarans, in: Proceedings of the 12th International Conference on Fast Sea Transportation, 2013.

[10] K. Zuercher, Experimental Waterjet Investiagtions, Ph.D. thesis, AMC, University of Tasmania (2015).

[11] K. Zuercher, N. Bose, J. Binns, G. Thomas, G. Davidson, Design and Commissioning Tests for Waterjet SelfPropulsion Testing of a MediumSpeed Catamaran Ferry using a Single Demihull, in: Proceedings of the Third International Symposium on Marine Propulsor, 2013.

[12] J. E. Rovere, Catamaran Resistance from Tests on a Single Demihull, in: Proceedings of International Conference on Fast Sea Transportation, 1997.

[13] F. M. White, Fluid Mechanics, McGraw-Hill Education - Europe, 2003.

[14] J. S. Kouh, Y. J. Chen, S. W. Chau, Numerical study on scale effect of form factor, Ocean Engineering 36.

[15] F. Stern, J. Yang, Z. Wang, M. Sadat-Hosseini, S. Bhushan, T. Xing, Computational ship hydrodynamics: Nowadays and way forward, International Shipbuilding Progress 60.

[16] L. Eca, M. Hoekstra, The numerical friction line, Marine Science and Technology 13.

[17] C. W. B. Grigson, A planar friction algorithm and its use in analysing hull resistance, Trans RINA.

[18] T. Katsui, H. Asai, Y. Himeno, Y. Tahara, The proposal of a new friction line, in: Fifth Osaka colloquium on advanced CFD applications to ship flow and hull form design , 2005.

[19] G. Hughes, Friction and form resisitance in turbulent flow and a proposed formulation for use in model and ship correlation, RINA 96. 
[20] T. Guiard, S. Leonard, F. Mewis, The Becker Mewis Duct Challenges in FullScale Design and new Developments for Fast Ships, in: Proceedings of the Third International Symposium on Marine Propulsor, 2013.

[21] J. M. Walker, M. P. Schultz, K. A. Flack, Skin-friction drag measurements on ship hull coating systems, in: Proceedings of 30th Symposium on Naval 640 Hydrodynamics, 2014.

[22] X. P. Tapia, Modelling of wind flow over complex terrain using OpenFoam, Master's thesis, University of Gavle (2009).

[23] H. Schlichting, Boundary Layer Theory, McGraw-Hill, 1979.

[24] D. Griggs, E. Woo, HSV-2 SWIFT Combined Standardization and Powering Trials Results, Tech. rep., Naval Surface Warfare Center, Carderock Division (2005).

[25] T. Oura, Y. Ikeda, Maneuverability of a Wavepiercing High-Speed Catamaran at Low Speed in Strong Wind, in: Proceedings of International Conference on Marine Research and Transportation, 2008.

[26] S. Schuster, Beitrag zur Frage der Kanalkorrektur bei Modellversuchen, Schiffstechnik.

[27] N. W. H. Bulten, Numerical Analysis of Waterjet Propulsion System, Ph.D. thesis, Eindhoven University of Technology (2006).

[28] J. de Cock, T. Van Beck, L. Muilwijk, R. Verbeek, J. Poelmann, Wartsila waterjets offer powerful and versatile propulsion solutions, Wartsila Technical Journal. 